\title{
Structural transformations in a simple-hexagonal Hg-Sn alloy under pressure
}

\author{
V. F. Degtyareva \\ Institute of Solid State Physics, Russian Academy of Sciences, Chernogolovka, Moscow district, 142432 Russia \\ O. Degtyareva, M. Winzenick, and W. B. Holzapfel \\ FB 6 Physik, Universität-GH-Paderborn, D-33095 Paderborn, Germany
}

(Received 18 May 1998)

\begin{abstract}
The structure of Sn alloyed with $\mathrm{Hg}$ (10 at. \%) was studied under pressure up to $66 \mathrm{GPa}$ with energydispersive $\mathrm{x}$-ray diffraction using synchrotron radiation. The ambient pressure simple hexagonal $(h P 1)$ phase transformed at $15 \mathrm{GPa}$ to a body-centered tetragonal (tI2) phase similar to the high-pressure forms of $\mathrm{Sn}$ and InBi. The axial ratio, $c / a$, of the $t I 2$ phase increased with pressure up to a maximum value of $c / a \approx 0.92$ at about $40 \mathrm{GPa}$ and remained nearly constant upon further compression to $55 \mathrm{GPa}$. At pressures above $55 \mathrm{GPa}$, a second phase transition, probably to an orthorhombic phase, was observed. The structural trends observed are discussed in terms of a Fermi-sphere-Brillouin-zone interaction. [S0163-1829(99)04709-8]
\end{abstract}

\section{INTRODUCTION}

Binary alloys of $\mathrm{Sn}$ with $\mathrm{Cd}, \mathrm{Hg}$, and In crystallize at ambient conditions in a simple-hexagonal $(h P 1)$ structure, ${ }^{1}$ later referred to as the $\mathrm{HgSn}_{9}$ structure type, ${ }^{2}$ with random occupation of the $1(a)$ site in $P 6 / \mathrm{mmm}$. The same structure has been found in more than ten Sn-based binary alloys by quenching from the liquid state. ${ }^{3}$ More recently, the $h P 1$ structure has also been obtained in other (non-Sn-based) alloys of main group metals by quenching from the liquid state (e.g., the In-Bi, In-Sb, Cd-Bi systems ${ }^{4}$ ) or by quenching from high pressure (e.g., the In-Bi, Pb-Sn, Al-Ge, Cd-Sb systems ${ }^{5}$ ). The $h P 1$ structure has also been observed in situ at high pressure in $\mathrm{Si}^{6,7} \mathrm{Ge},{ }^{8}$ and in the binary alloy systems Al-Ge, ${ }^{9} \mathrm{Zn}-\mathrm{Sb}$, and $\mathrm{Cd}-\mathrm{Sb} .{ }^{10}$

In the lighter group IV elements $\mathrm{Si}$ and $\mathrm{Ge}$, the $h P 1$ structure appears under pressure after the white tin ( $t I 4)$ structure as one of the intermediate structures ${ }^{11}$ which are transformed at even higher pressures into one of the closepacked structures $h P 2, c F 4$, or $h P 4$. $^{6,8,12}$ The heavier element $\mathrm{Sn}$, however, shows a different structural sequence under pressure with transitions from white tin (tI4) to bodycentered tetragonal $(t I 2)$ and to body-centered-cubic ( $c I 2)$ structures. $^{13,14}$

The axial ratio $(0.91<c / a<0.95)$ observed for the $t I 2$ structure of $\mathrm{Sn}$ is distinct among those elements exhibiting this structure. $c / a$ ratios of $0.71(\mathrm{Hg}), 0.82(\mathrm{~Pa})$, and $c / a$ $>1.0(\mathrm{In}, \mathrm{Ga}, \mathrm{Ce}, \mathrm{Pu})$ have been reported for other elements when in $t I 2$ structure. ${ }^{15}$ Only recently, in $\mathrm{InBi}$ at high pressure, ${ }^{16}$ has a $t I 2$ structure been reported with $c / a$ values similar to the ones of the high-pressure phases of Sn.

The $h P 1$ structure occurs only for the lighter group IV elements $\mathrm{Si}$ and $\mathrm{Ge}$ at high pressure. The heavier group IV elements $\mathrm{Sn}$ and $\mathrm{Pb}$ do not exhibit this structure, and furthermore show a common tendency to transform to a $c I 2$ structure at the highest pressures reached to date, rather than to a closed-packed structure like $\mathrm{Si}$ and $\mathrm{Ge}$. These differences in behavior suggest that interesting structural effects may occur in $\mathrm{Sn}-\mathrm{Hg}$ alloys related to their $h P 1$ ambient pressure struc- ture. In this paper, the structure of $\mathrm{HgSn}_{9}$ is studied under pressure.

\section{EXPERIMENTAL DETAILS}

High-pressure x-ray-diffraction experiments were executed on samples contained in diamond anvil cells. ${ }^{17}$ Samples were placed into gasket holes of 100 to $200 \mu \mathrm{m}$ diameter along with ruby or gold as a pressure marker. ${ }^{18,19}$ Mineral oil was normally used as the pressure transmitting medium. The diffraction experiments were performed in the energy-dispersive mode (EDXD) using synchrotron radiation at the beam line F3 in HASYLAB, DESY (Hamburg). ${ }^{20-22}$

The sample material was prepared by melting a 1:9 (Hg:Sn) mixture of the pure elements $(99,99 \%)$ in an evacuated silica tube. Diffraction data obtained with a diffractometer (SIEMENS-D500) confirmed the occurrence of a pure $h P 1$ phase at ambient conditions with lattice parameters $a$ $=320.8(1) \mathrm{pm}$ and $c=298.5(2) \mathrm{pm}$. These values correspond to an alloy composition with 10 at. $\% \mathrm{Hg}^{2}$ Small pieces from this sample were used for three different sets of experiments. All pressure experiments were performed at room temperature. Specially adapted software ${ }^{23}$ was used for the evaluation of the diffraction spectra.

\section{EXPERIMENTAL RESULTS}

Upon compression, the pure $h P 1$ phase is observed up to 13.7 GPa. Beginning at this pressure, diffraction peaks corresponding to the next phase are observed and increase in intensity at the expense of the original $h P 1$ diffraction peaks. Above $19 \mathrm{GPa}$, only is phase is present. Peak indexing indicates that the phase possesses a tetragonal bodycentered structure with two atoms per unit cell ( $t I 2)$ and space group $I 4 / \mathrm{mmm}$. The phase transition is reversible with a hysteresis of about $7 \mathrm{GPa}$. The $h P 1$ phase is recovered completely on decompression below $12 \mathrm{GPa}$. This observation indicates that the alloy does not decompose under pressure. Typical diffraction spectra at pressures near 


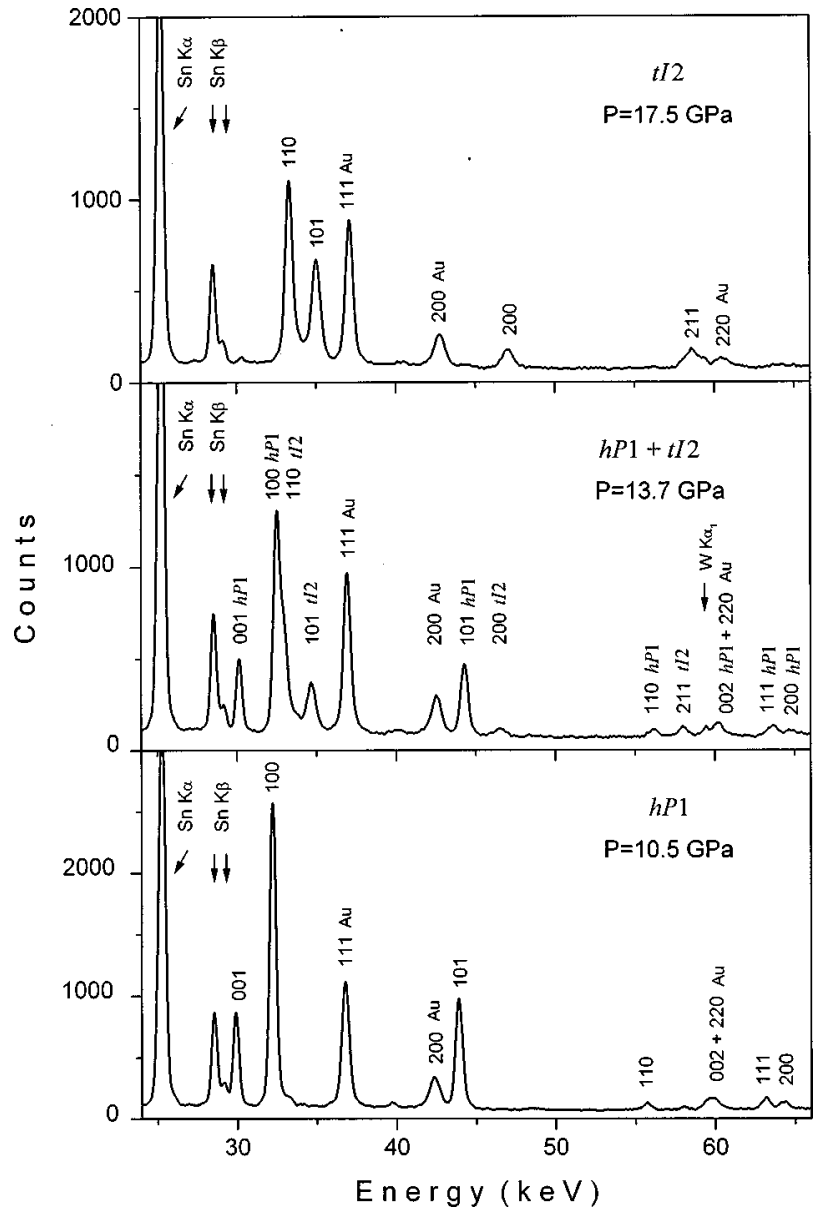

FIG. 1. EDXD spectra of $\mathrm{HgSn}_{9}$ at different pressures measured with $2 \theta=8.36^{\circ}$. Fluorescence peaks from $\mathrm{Sn}$ and $\mathrm{W}$ are marked by arrows. Diffraction peaks of phase I (below) are indexed according to the $h P 1$ structure and phase II (above) according to the $t I 2$ structure.

the phase transition are shown in Fig. 1. The use of a larger diffraction angle $\left(2 \theta=10.2^{\circ}\right)$ allowed us to observe up to ten lines for the phase and supported the assignment of a $t I 2$ structure (Table I). All observed $d$ spacings $d_{\text {obs }}$ can be as-

TABLE I. ( $h k l)$ indices, observed and calculated $d$ spacings, together with observed and calculated intensities for the EDXD spectrum of $\mathrm{HgSn}_{9}$ (II) at $19.3 \mathrm{GPa}$ obtained at $2 \theta=10.2^{\circ}$. The best fit gives $a=360.6(1) \mathrm{pm}$ and $c=328.4(2) \mathrm{pm}$ for the $t I 2$ structure (space group $I 4 / \mathrm{mmm}$ ). The intensities are calculated for a randomly disordered alloy with a sample thickness of $40 \mu \mathrm{m}$.

\begin{tabular}{lccrr}
\hline \hline$(h k l)$ & $d_{\text {obs }}(\mathrm{pm})$ & $d_{\text {calc }}(\mathrm{pm})$ & $I_{\text {obs }}$ & $I_{\text {calc }}$ \\
\hline 110 & 254.7 & 255.0 & 93 & 58 \\
101 & 243.1 & 242.8 & 100 & 100 \\
200 & 180.5 & 180.3 & 20 & 13 \\
002 & 164.0 & 164.2 & 2 & 5 \\
211 & 144.8 & 144.8 & 20 & 20 \\
112 & 138.0 & 138.0 & 4 & 8 \\
220 & 127.5 & 127.5 & 2 & 3 \\
202 & 121.8 & 121.4 & 3 & 4 \\
310 & 114.3 & 114.0 & 3 & 3 \\
301 & 113.2 & 112.9 & 3 & 3 \\
\hline \hline
\end{tabular}

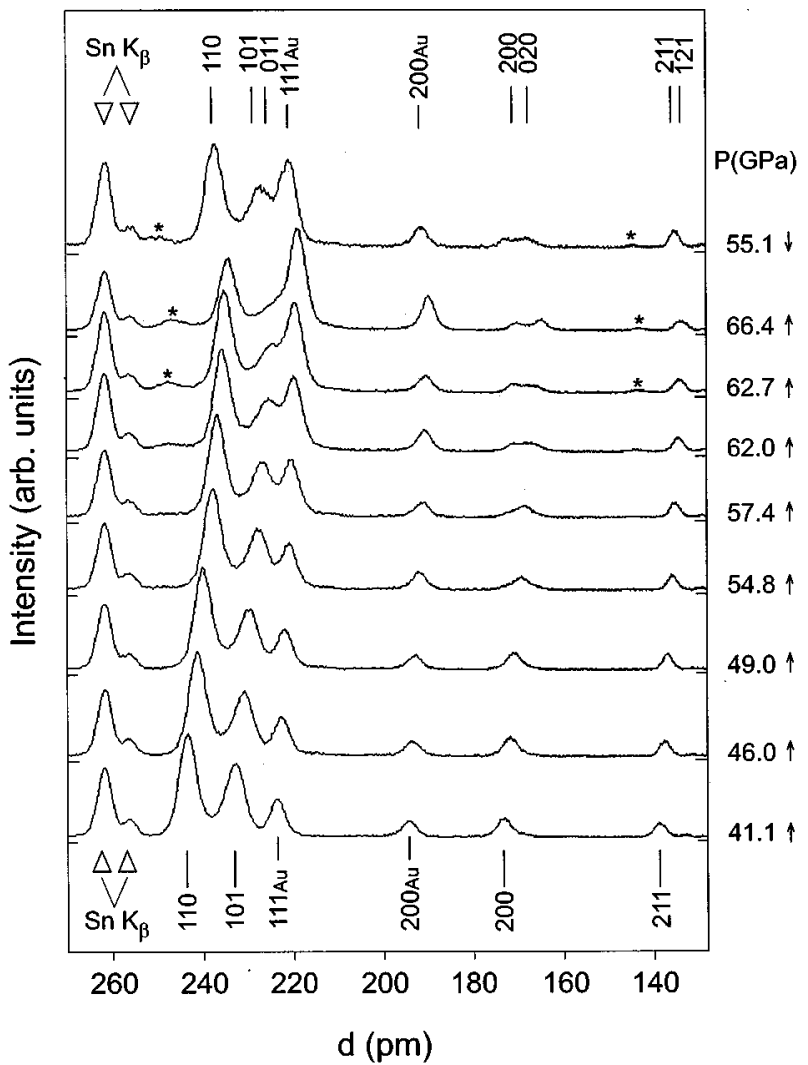

FIG. 2. Effect of pressure on $\mathrm{HgSn}_{9}$ and Au diffraction peaks in EDXD spectra $\left(2 \theta=9.52^{\circ}\right)$ showing the splitting of diffraction peaks denoted by orthorhombic indexing and additional features marked by asterisks.

signed to the calculated values $d_{\text {calc }}$ of the $t I 2$ structure within the limits of $\pm 0.4 \mathrm{pm}$ typical for the present technique. The observed and calculated intensities, $I_{\mathrm{obs}}$ and $I_{\text {calc }}$, show very reasonable agreement if one takes into account the problem of texture associated with the present technique. ${ }^{24}$ Only a single, weak, unassignable line near $40 \mathrm{keV}$ was observed. The line was present in the spectra of both the $h P 1$ and $t I 2$ phases (Fig. 1) and could possibly result from a small admixture of another intermediate phase, whose structure seems to be unknown. ${ }^{25}$

Since most of the high-pressure measurements were taken with a diffraction angle $2 \theta=9.52^{\circ}$, as illustrated in Fig. 2, only the four most intense Bragg peaks [(110), (101), (200), and (211)] of the $\mathrm{HgSn}_{9}$ structure (tI2) were used in the determination of lattice parameters to have comparable data sets. Differences in the evaluation of these reduced data sets, relative to full fits of ten lines, do not exceed the statistical error of $\pm 0.2 \mathrm{pm}$. However, due to nonhydrostaticity, ${ }^{26}$ the positions of some peaks can deviate from the calculated values by up to $\pm 0.4 \mathrm{pm}$.

The spectra for the region of two-phase coexistence give the most reliable values for the volume change at the transition. From the spectrum at $13.7 \mathrm{GPa}$ (Fig. 1), one obtains coordination number increases from $2+6$ to $8+2+4$. At the same time, the corresponding interatomic distances also increase from $d_{1}(2)=c=282.2 \mathrm{pm}$ and $d_{2}(6)=a=302.7 \mathrm{pm}$ to $\quad d_{1}(8)=307.0 \mathrm{pm}, \quad d_{2}(2)=c=331.4 \mathrm{pm}, \quad d_{3}(4)=a$ $=365.5 \mathrm{pm}$ (with $\Delta d= \pm 0.2 \mathrm{pm}$ ). In total, the atomic volume decreases by only $1.1(2) \%$ at the transition. 


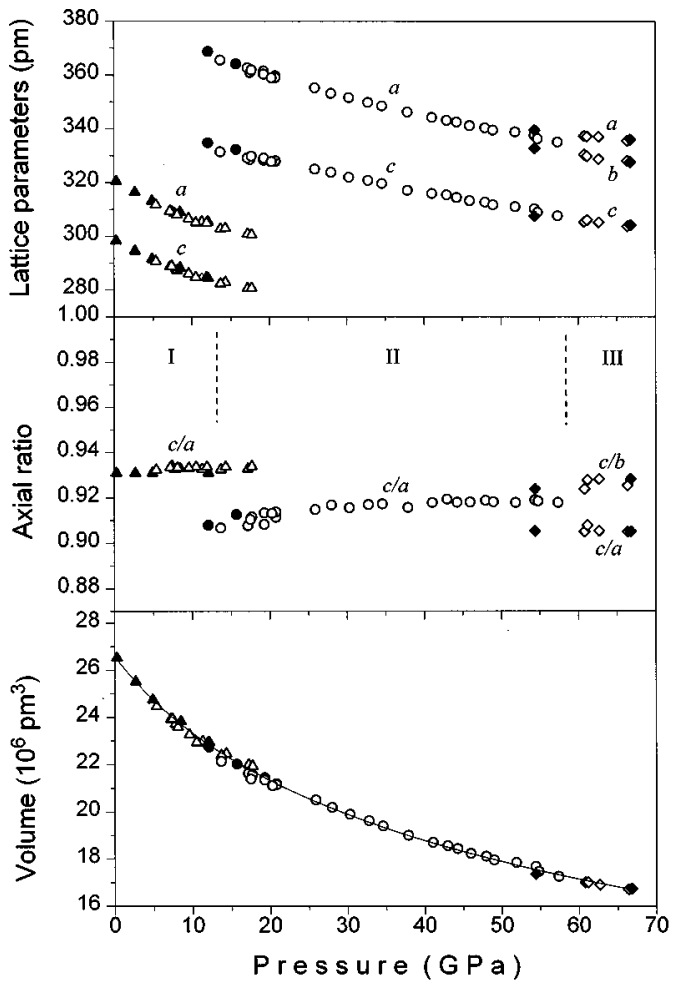

FIG. 3. Pressure dependence of the lattice parameters, the $c / a$ ratio and the atomic volume of $\mathrm{HgSn}_{9}$. Triangles: phase I ( $\left.h P 1\right)$. Circles: phase II ( $t I 2)$. Diamonds: phase III. Open symbols correspond to data taken on increasing pressure and solid symbols on decreasing pressure, respectively.

Above $55 \mathrm{GPa}$, a splitting of some of the $t I 2$ diffraction lines was noticed, as illustrated most clearly in Fig. 2 for the (200) $-t I 2$ peak. A tentative indexing of the pattern at 66.4 GPa based on an orthorhombically distorted lattice explains all the dominant peaks. Two additional weak features, however, are also noticed and indicated in Fig. 2 with asterisks. Possibly, these features result from minor unknown impurities. However, if they belong to phase III, the structure of this phase would have to be more complex, but closely related to the body-centered-orthorhombic cell.

A strong hysteresis in the II-III transition was observed, as evidenced by the top spectrum in Fig. 2 and the solid diamond data points in Fig. 3, and suggests a first-order character for this transition. A continuous increase of the orthorhombic distortion at the transition, however, cannot be definitively excluded. Therefore, the stated values for the axial ratios $c / b$ and $c / a$ of phase III in the "mixed-phase region" depend on the interpretation of the spectra.

We note further that the assignment of a body-centeredorthorhombic structure for phase III results in a smooth continuation of the equation of state (EOS) given in the lower part of Fig. 3. The corresponding pressure dependencies of the lattice parameters and their ratios for all three phases are illustrated in Fig. 3. The axial ratio $c / a$ of the $h P 1$ phase increases slightly with pressure from 0.930 at ambient pressure to 0.934 at $17.7 \mathrm{GPa}$, the highest pressure at which this phase was observed. The $t I 2$ phase appears at $13.7 \mathrm{GPa}$ with an axial ratio 0.907 . With increasing pressure, $c / a$ initially increases and levels off at a maximum value of 0.919 at about $40 \mathrm{GPa}$. No further change was observed up to about $55 \mathrm{GPa}$, the pressure at which the next transition occurs.

The average atomic volume of all three phases can be represented in a first approximation by one common equation of state (EOS) as illustrated in Fig. 3, since the volume discontinuities at both phase transitions are less than $2 \%$. With the given volume $V_{0}=26.6 \times 10^{6} \mathrm{pm}^{3}$ at ambient conditions, the fitted curve in Fig. 3 corresponds to the parameter values $K_{0}=59(1) \mathrm{GPa}$ and $K_{0}^{\prime}=3.9(1)$ for the bulk modulus and its pressure derivative at ambient conditions, when one uses either an EOS of the second-order Birch type $^{27}$ or the more recently proposed type AP $2 .{ }^{28}$

\section{DISCUSSION}

The present high-pressure study of an Sn-Hg alloy with $h P 1$ structure reveals a transition to the $t I 2$ structure, in contrast to the behavior of the light group IV elements Si and Ge where the high-pressure $h P 1$ phase has the tendency to transform at higher pressures directly or indirectly into one of the close-packed structures. However, some similarities with the high-pressure behavior of the heavier element $\mathrm{Sn}$ and of the heavier III-V compound InBi can be noticed with respect to the occurrence of the $t I 2$ structure. ${ }^{14,16}$ Both the $h P 1$ and the $t I 2$ structures are characterized by special values of $c / a$ and it is interesting to examine possible causes for these special values.

The $h P 1$ structure appears in the light group IV elements $\mathrm{Si}$ and $\mathrm{Ge}$ under pressure in their metallic forms and in alloys with an average valence electron number of nearly four. This structure is characterized in all cases by a value of $c / a$ in the range between 0.92 and 0.95 . An explanation for this specific value was given by Weaire and Williams ${ }^{29}$ using simple symmetry arguments for both real and reciprocal spaces. Because both the real and reciprocal lattices are hexagonal, it was argued that the electrostatic energy is minimized by a balance between contributions from effects related to the real and reciprocal lattices, respectively, with the requirement that $c / a \approx c^{*} / a^{*}$. This requirement leads to $c / a=(\sqrt{ } / 2)^{1 / 2}$ $=0.931$, in close agreement with previously observed experimental data and similar to the present value for $\mathrm{HgSn}_{9}(h P 1)$ given in Fig. 3.

The $t I 2$ structure observed in the $\mathrm{Sn}-\mathrm{Hg}$ alloy is related by a slight distortion to the $c I 2$ structure which occurs as a high-pressure structure for $\mathrm{Sn}$ and $\mathrm{InBi}$. For all these structures, similar variations of $c / a$ with pressure were found: a slight increase with pressure initially and then an approach towards a nearly constant value over an extended pressure range. The observed upper value of $c / a$ is 0.96 for $\mathrm{Sn}$ and $\mathrm{InBi}$, but only 0.92 for $\mathrm{HgSn}_{9}$, a result that suggests that the degree of distortion $(c / a)$ is determined by the average number of valence electrons $n$ (electron/atom) or, in other words, by the valence electron concentration.

The earlier experimental observations of the $t I 2$ structure in Sn under pressure have stimulated theoretical studies. These studies have predicted only small differences in the crystal structure energy between the $t I 2$ and $c I 2$ phases $^{30}$ as well as a $c / a$ value of 0.90 for the $t I 2$ structure and the pressure of $46 \mathrm{GPa}$ for the $t I 2-c I 2$ transition. ${ }^{31}$ Semiempir- 


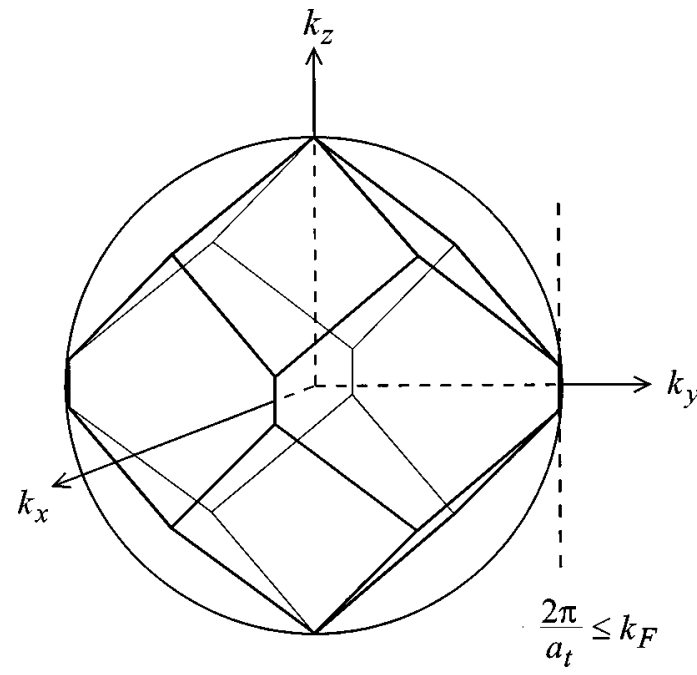

FIG. 4. The Brillouin zone with (110) and (101) planes and the Fermi sphere for an electron concentration of 3.8 for a bodycentered-tetragonal ( $t I 2)$ structure with $c / a=0.92$ (the case of $\mathrm{HgSn}_{9}$ ).

ical calculations for tin under pressure ${ }^{32}$ predict that $c / a$ in $\operatorname{Sn}(t I 2)$ should not exceed $\approx 0.95$ and would have to change discontinuously to $c / a=1$ at the transition to $\operatorname{Sn}(c I 2)$.

Experimental observations of stronger distortions of $c / a$ in the $t I 2$ structure for lower electron concentrations further suggest the relevance of the concept of Brillouin-zoneFermi-sphere interaction. ${ }^{33}$ Within the simple nearly-freeelectron model, consideration of a matching between the Brillouin zone and Fermi sphere explain qualitatively special values in certain lattice parameter relations for noncubic (hexagonal or tetragonal) structures, ${ }^{34}$ including the formation of the $t I 2$ structure in simple metals with nearly four valence electrons.

For a $c I 2$ structure with the atomic volume $V=a^{3} / 2$, one obtains for the ratio of the Fermi-sphere radius, $k_{F}$ $=\left(3 \pi^{2} n / V\right)^{1 / 3}$, to the lattice parameter in $k$ space, $2 \pi / a$, at $n=4$ the value $k_{F} /(2 \pi / a)=(3 / \pi)^{1 / 3}<1$. This means that $k_{F}$ $<2 \pi / a$ or, in other words, the (110) planes of the Brillouin zone are intersecting the Fermi sphere near the corners of the zone. A Brillouin-zone-Fermi-sphere interaction can therefore favor a tetragonal distortion as illustrated in Fig. 4.

This model allows us to estimate the upper value of $c / a$ for the $t I 2$ structure from the condition $2 \pi / a_{t} \leqslant k_{F}$, which gives $c / a \leqslant 3 / 4 \pi \cdot n$, or $c / a \leqslant 0.96$ for $n=4$ (Sn, InBi) and $c / a \leqslant 0.91$ for $n=3.8\left(\mathrm{HgSn}_{9}\right)$, in good agreement with the experimentally observed values in Fig. 5. This model indicates that tetragonal distortions would occur when the electron concentrations $n<4.2$. In fact, the few experimental results for $\mathrm{SnSb}$ (Ref. 36) and $\mathrm{SnBi}$ (Ref. 37) with $n=4.5$ show only cubic structures under pressure and no tetragonal distortions. In addition, the group $\mathrm{V}$ elements $\mathrm{As}, \mathrm{Sb}$, and $\mathrm{Bi}$ $(n=5)$ are known to transform into $c I 2$ under pressure

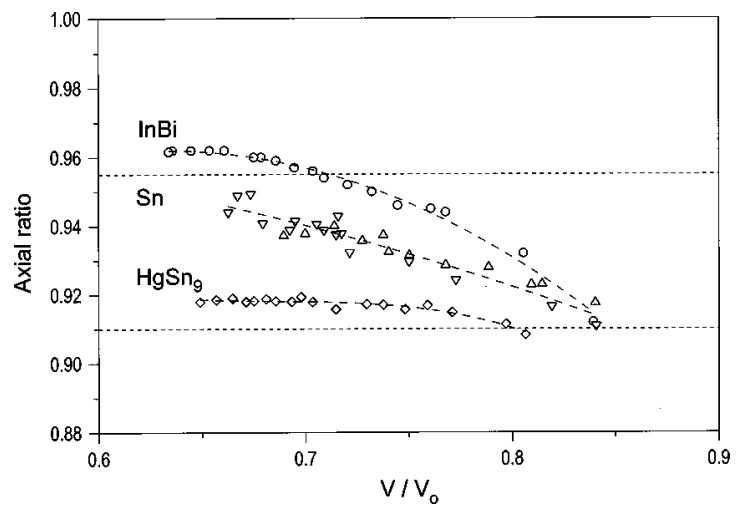

FIG. 5. Comparison of $c / a$ ratios vs $V / V_{0}$ for the $t I 2$ structures: $\mathrm{HgSn}_{9}$ (present work), Sn ( $\Delta$ is data from Ref. $14, \nabla$ is data from Ref. 35), and InBi (data from Ref. 16). The corresponding values for $V_{0}$ are, respectively, $26.6 \times 10^{6} \mathrm{pm}^{3}$ for $\mathrm{HgSn}_{9}(h P 1), 27.0$ $\times 10^{6} \mathrm{pm}^{3}$ for tin $\left(\beta\right.$-Sn) and $28.0 \times 10^{6} \mathrm{pm}^{3}$ for InBi (metallic InBi II). Special values of $c / a$ for $\operatorname{InBi}$ and $\mathrm{Sn}(c / a=0.96)$ and for $\mathrm{HgSn}_{9}(c / a=0.91)$ are represented by dashed lines (see text).

via a complicated intermediate structure ${ }^{28}$ without a transition through $t I 2$.

The $t I 2-c I 2$ transition under pressure in $\mathrm{Sn}$ and also (probably) in InBi represents a trend to higher symmetry, which is expected from an increase in the electrostatic energy. However, the first-order character of this transition, as evidenced by the discontinuity in $c / a$ can be understood only by detailed considerations of the band-structure energy and the Brillouin-zone-Fermi-sphere interaction. In contrast to these two examples, the $t I 2$ phase of $\mathrm{HgSn}_{9}$ is not transformed directly into a cubic phase at higher pressures, but probably to an orthorhombic phase. A possible explanation for this unique behavior may be related to the lower value of the valence electron concentration and to the correspondingly lower limiting value for $c / a$, which would require a larger jump at the transition to $c I 2$. Since an orthorhombic distortion would result in a splitting of $q_{200}$ in the reciprocal lattice, the reciprocal-lattice vectors $q_{200}$ and $q_{020}$ could adopt values corresponding to $q_{200}<2 k_{F}<q_{020}$, which still represents a situation with a strong Brillouin-zone-Fermisphere interaction.

From the present results, one can expect that many other binary alloys with valence electron concentrations close to 4 should follow the same structural sequence under pressure including an intermediate $t I 2$ structure and a $c I 2$ phase at higher pressures.

\section{ACKNOWLEDGMENTS}

The authors wish to thank H.-J. Hesse and W. Bröckling for technical assistance and $\mathrm{K}$. Bray for critical reading and comments. O.D. is grateful for a grant from the FriedrichEbert-Stiftung and financial support by the German Ministry of Research and Technology (BMBF) under Contract No. 05647 PPA is also gratefully acknowledged. 
${ }^{1}$ G. V. Raynor and J. A. Lee, Acta Metall. 2, 616 (1954).

${ }^{2}$ G. C. Che, M. Ellner, and K. Schubert, J. Mater. Sci. 26, 2417 (1991).

${ }^{3}$ R. H. Kane, B. C. Giessen, and N. J. Grant, Acta Metall. 14, 605 (1966); It should be noted that the results of this study are compiled by P. Villars, in Pearson's Handbook Desk Edition (Materials International Society, Materials Park, Oh, 1997) with different structural assignments.

${ }^{4}$ B. C. Giessen, Adv. X-Ray Anal. 12, 23 (1969).

${ }^{5}$ E. G. Ponyatovskii and V. F. Degtyareva, High Press. Res. 1, 163 (1989).

${ }^{6}$ H. Olijnyk, S. K. Sikka, and W. B. Holzapfel, Phys. Lett. 103A, 137 (1984).

${ }^{7}$ J. Z. Hu and I. L. Spain, Solid State Commun. 51, 263 (1984).

${ }^{8}$ Y. K. Vohra, E. Brister, S. Desgreniers, A. L. Ruoff, K. L. Chang, and M. L. Cohen, Phys. Rev. Lett. 56, 1944 (1986).

${ }^{9}$ V. F. Degtyareva, F. Porsch, E. G. Ponyatovskii, and W. B. Holzapfel, Phys. Rev. B 53, 8337 (1996).

${ }^{10}$ V. F. Degtyareva, I. Bdikin, and S. S. Khasanov, Fiz. Tverd. Tela (St. Petersburg) 39, 1509 (1997) [Phys. Solid State 39, 1341 (1997)].

${ }^{11}$ M. I. McMahon and R. J. Nelmes, Phys. Status Solidi B 198, 389 (1996).

${ }^{12}$ S. J. Duclos, Y. K. Vohra, and A. L. Ruoff, Phys. Rev. Lett. 58, 775 (1987).

${ }^{13}$ J. D. Barnett, V. E. Bean, and H. T. Hall, J. Appl. Phys. 37, 875 (1966).

${ }^{14}$ H. Olijnyk and W. B. Holzapfel, J. Phys. Colloq. 45, C8-153 (1984).

${ }^{15}$ P. Villars and L. D. Calvert, Pearson's Handbook of Crystallographic Data for Intermetallic Phases (American Society for Metals, Metals Park, Oh, 1985).

${ }^{16}$ V. F. Degtyareva, M. Winzenick, and W. B. Holzapfel, Phys. Rev. B 57, 4975 (1998).

${ }^{17}$ K. Syassen and W. B. Holzapfel, Europhys. Conf. Abstr. 1A, 75 (1975).
${ }^{18}$ H. K. Mao, P. M. Bell, J. W. Shaner, and D. J. Steinberg, J. Appl. Phys. 49, 3276 (1978).

${ }^{19}$ D. L. Heinz and R. Jaenloz, J. Appl. Phys. 55, 4 (1984).

${ }^{20}$ W. B. Holzapfel, in High Pressure Chemistry, edited by H. Kelm (Reidel, Dordrecht, 1978), p. 177.

${ }^{21}$ W. A. Grosshans, E.-F. Düsing, and W. B. Holzapfel, High Temp.-High Press. 16, 539 (1984).

${ }^{22}$ J. W. Otto, Nucl. Instrum. Methods Phys. Res. A 384, 552 (1997).

${ }^{23}$ F. Porsch, EDXPOWDER, program for evaluation of EDXD spectra, RTI, Paderborn, Germany (1996).

${ }^{24}$ H. W. Neuling, O. Schulte, T. Krüger, and W. B. Holzapfel, Meas. Sci. Technol. 3, 170 (1992).

${ }^{25} \mathrm{M}$. Hansen and K. Anderko, Constitution of Binary Alloys (McGraw Hill, New York, 1958), reprinted by Genium Publishing Corp., New York (1989).

${ }^{26}$ A. K. Singh and G. C. Kennedy, J. Appl. Phys. 45, 4686 (1974).

${ }^{27}$ F. Birch, J. Geophys. Res. 83, 1257 (1978).

${ }^{28}$ W. B. Holzapfel, High Press. Res. 16, 81 (1998).

${ }^{29}$ D. Weaire and A. R. Williams, Philos. Mag. 19, 162 (1969); 19, 1105 (1969).

${ }^{30}$ J. L. Corkill, A. Garcia, and M. L. Cohen, Phys. Rev. B 43, 9251 (1991).

${ }^{31}$ B. H. Cheong and K. J. Chang, Phys. Rev. B 44, 4103 (1991).

${ }^{32}$ N. E. Christensen and M. Methfessel, Phys. Rev. B 48, 5797 (1993).

${ }^{33} \mathrm{H}$. Jones, The Theory of Brillouin Zones and Electronic States in Crystals (North-Holland, Amsterdam, 1962).

${ }^{34}$ V. F. Degtyareva, in Stability of Materials, NATO ASI Series B Physics, edited by A. Gonis, P. E. A. Turchi, and J. Kudrnovsky (Plenum, New York, 1996), Vol. 355, p. 465.

${ }^{35}$ M. Liu and L. Liu, High Temp.-High Press. 18, 79 (1986).

${ }^{36}$ V. G. Losev, S. S. Kabalkina, and L. F. Vereshchagin, Fiz. Tverd. Tela (Leningrad) 12, 2942 (1970) [Sov. Phys. Solid State 12, 2374 (1970)].

${ }^{37}$ V. F. Degtyareva, I. Bdikin, and S. S. Khasanov, Solid State Commun. 99, 907 (1996). 